\section{Facets of negative affectivity and blood pressure in middle-aged men}

\section{Cornel V. Igna, Juhani Julkunen, Jari Lipsanen, Hannu Vanhanen \\ The Finnish Heart Association, Helsinki Heart District, University of Helsinki, Finland}

\section{Abstract}

Research results suggesting that facets of negative affectivity, i.e. anxiety, anger-hostility, and depression, relate to incident cardiovascular diseases have been steadily increasing. Evidence for depression has been especially extensive. Elevated blood pressure, a major risk factor of cardiovascular diseases, is one probable mediator in this context. The purpose of this study was to clarify the relationship of specific key elements of depressive disposition, i.e. depressive symptoms, hopelessness and vital exhaustion, with health behavior and blood pressure. Study sample was comprised of 710 middle-aged men. Participants completed self-report questionnaires assessing health behavior, depressive symptoms, vital exhaustion and hopelessness. Statistical analyses involved descriptive analyses, correlations and path analysis. Depressive symptoms and vital exhaustion associated with several unfavorable lifestyles such as smoking, alcohol consumption, and inactivity (standardized solution coefficients: $0.10,0.14,0.17$, accordingly). However, no significant direct associations with blood pressure could be found for depressive symptoms or vital exhaustion. Hopelessness associated only with unhealthy diet (standardized solution coefficient -0.10) Moreover, for hopelessness, results showed a direct but inverse association with systolic blood pressure (standardized solution coefficient -0.08 ). Results suggest that the previously reported relations of depression and vital exhaustion with blood pressure could be mediated by unfavorable lifestyles. The relation of hopelessness with adverse health behaviors seems to be less significant. Also, the role of hopelessness as a risk factor of elevated blood pressure is not supported by the results of this study.

\section{Introduction}

Negative affectivity (NA) has been conceptualized as a higher order construct including three main affective dispositions, i.e. depres- sion, anxiety, and anger-hostility. ${ }^{1}$ Several studies have shown that all three dispositions confer an elevated risk of cardiovascular diseases (CVD), and extensive evidence supports the role of depression as a risk factor of CVD.1,2 At the same time, however, depression has been operationalized in several different ways including related concepts such as vital exhaustion and hopelessness. ${ }^{3,4}$ The diversity as well as the overlap of these constructs and measurements is leading to problems for theory testing and for interpretation of empirical results. So far, these questions have remained unsolved as in most of the empirical work in this area only a single indicator of depression is used at a time. ${ }^{1}$

Another important issue is the question about pathways between depressive disposition and CVD. Elevated blood pressure (BP), as a well-established, major risk factor of CVD is one plausible mediating mechanism. In this study we focus on comparing three indicators of depressive disposition, i.e. depressive symptoms, hopelessness and vital exhaustion, and try to clarify their relations with blood pressure.

Recently, there has been an increasing effort to investigate in more detail the possible mediating mechanisms between depressive disposition and CVD, including the role of blood pressure. A widely supported theory explains the relation of NA with poor vascular health by assuming that high levels of negative feelings activate the sympathetic-adrenalmedullary system and hypothalamic-pituitaryadrenal-cortical axis system, followed by increased serum levels of catecholamines and cortisol which have an influence on BP and heart rate. ${ }^{5}$ Also, other different mediating mechanisms that involve health behavior, ${ }^{6}$ neurotransmitters activity or a genetic vulnerability have been discussed.7

Other unclear aspects are the depressionhopelessness and depression-vital exhaustion relations. Hopelessness is defined as overall negative expectations about one's personal future. However, while hopelessness is included in the definition of depression, 8 the association of hopelessness with known depression scales is not very strong, and the presence of hopelessness is not necessarily related to depression suggesting that hopelessness could be to some extent a different concept from depression.4,9 From this point of view the results presented by Everson et al. ${ }^{10}$ are especially interesting; they found that hopelessness but not depression, predicted incident hypertension in middle-aged men.

Moreover, although it has been agreed that depression and vital exhaustion (VE) are related, there still is an ongoing debate on whether to consider them as overlapping or distinct concepts. Furthermore, vital exhaustion has been found to precede depression, or to repre-
Correspondence: Juhani Julkunen, Institute of Behavioural Sciences, University of Helsinki, PO Box 9 (Siltavuorenpenger 1A), FIN-00014, Finland.

Tel.: +358.919129503 - Fax: +358.919129521

E-mail: juhani.julkunen@helsinki.fi

Key words: blood pressure, health behavior, vital exhaustion, depression, hopelessness.

Acknowledgments: we gratefully acknowledge the contribution of Helsinki Heart District personal in collecting the data. The main study was funded by the Finnish Slot Machine Corporation.

Contributions: CI has designed the study questions and conducted the statistical analyses of the data, and drafted the article; $\mathrm{JJ}$ is responsible for the design of psychosocial assessments, and has participated in revising the article; JL is responsible for designing and conducting the mediation analyses as well as reporting results of statistical analyses, and he has participated in revising the article; HV drafted the parts of the article demanding medical expertise, and he has participated in revising the manuscript.

Conflict of interests: the authors declare no potential conflicts of interests.

Received for publication: 4 November 2012.

Revision received: 16 December 2012

Accepted for publication: 17 December 2012

This work is licensed under a Creative Commons Attribution NonCommercial 3.0 License (CC BYNC 3.0).

(C) Copyright C.V. Igna et al., 2013

Licensee PAGEPress, Italy

Health Psychology Research 2013; 1:e14 doi:10.4082/hpr.2013.e14

sent a broader concept than depression. ${ }^{11}$ Some studies have indicated that depression and vital exhaustion associate differently to coronary disease risk factors but again, 12 in another study no significant differences were found. 13

While the impact of depression on life-style factors is satisfactorily understood, the role of vital exhaustion or hopelessness is not so clear. There are studies that suggest a positive relation between vital exhaustion and smoking, ${ }^{14}$ alcohol consumption and inactivity, ${ }^{15,16}$ but in some other studies no relations of vital exhaustion with these variables were found. 17 Moreover, we could not find any article that had explored the relation between vital exhaustion and diet.

There are several previous studies suggesting that depressed or hopeless persons are more likely to be engaged in smoking, in sedentary lifestyle and alcohol over-consump- 
tion. ${ }^{6}$ For example, Joyner-Grantham et al. ${ }^{18}$ concluded that hopelessness could explain why, in spite of adequate hypertension healthrelated knowledge, patients were not improving their lifestyles.

Taken together, a major limitation in previous literature is that only a few studies - if any - have assessed more than one depressionrelated factor, health behavior, and clinical outcomes at the same time. Hypothetical relations between all these above mentioned groups of variables are summarized in the model illustrated in Figure 1.

This basic model was used to test the direct and indirect effects of depression, hopelessness and vital exhaustion on blood pressure. A related model has been used to test the relation between ways of anger expression and blood pressure. 19

Furthermore, because this model is a hypothetical one it does not include any assumption that those three depression-related concepts would be working in the same way or not. The indirect link assumes that depression/hopelessness/vital exhaustion relate to blood pressure through life-style factors. In this context, smoking, alcohol and coffee consumption, diet, and exercise habits are considered as the most important lifestyle mediators. The previously established strong relations between lifestyle variables and body mass index and, between body mass index and blood pressure, suggest that body mass index is an important mediating factor between lifestyle and blood pressure. Education, age and marital status were controlled for as confounding variables. Education was assumed to confound the relation of depression/hopelessness/vital exhaustion with lifestyle factors. In sum, the aim of the present study was to investigate the possible direct or indirect relationships of depressive symptoms, hopelessness and vital exhaustion with blood pressure.

\section{Materials and Methods}

\section{Design and procedures}

The present study is a sub-study of the Helsinki Metabolic Syndrome Prevention Trial, which was an uncontrolled preventive trial aimed at improving prevention of metabolic syndrome, type 2 diabetes and cardiovascular diseases. The goals were to screen and find men with a cluster of cardiovascular risk factors, and to offer them individual counseling.

An invitation letter with questionnaires was mailed to 2990 middle-aged, male Helsinki citizens. It was a population sample, all men of the age-cohorts of 40-, 45-, 50-, and 55-years living in the North-Eastern district of the city of Helsinki were invited to participate. Of them, 1288 (43.1\%) participated in a screening visit and were asked to answer questionnaires dealing with lifestyle factors and psychosocial risk factors of CVD. Study nurses from the Helsinki Heart District interviewed all participants and recorded basic medical measurements. Evaluation of risk factors has been described in detail elsewhere. ${ }^{19}$

Data collection was carried out between May 2001 and June 2004. The Ethical Committee of the Helsinki University Central Hospital (HUS) approved the study on the 24th April 2001.

\section{Participants}

A total of 1288 men from age cohorts of 40 , 45,50 , and 55 years were screened for the preventive trial. From the total sample, all those with age below 45 years (22.9\%) were excluded from the present analysis because due to logistic reasons complete psychosocial data was available only for high-risk cases in this cohort. From the remaining sample $(\mathrm{N}=1005)$ were excluded those with missing depression, hopelessness or vital exhaustion scores, those with unclear smoking status and those with blood pressure medication. The final study sample was 710 men; mean age was 49.41 years (S.D. 3.78). Using the 4-step classification of Beck Depression Inventory (cut-off values: 0-9: no depression, 10-18: mild depression, 19-29: moderate depression, 30-60: severe depression), $76.1 \%$ of the subjects had no depression, $16.9 \%$ had mild depression, $5.8 \%$ had moderate depression, and only $1.3 \%$ had symptoms of severe depression. In the study sample thirteen persons had diabetes and ten persons had angina pectoris and/or symptoms of coronary heart disease.

\section{Measures}

In the present sub-study we used three types of variables: socio-demographic (age, education, marital status); self-report measures (lifestyle risk factors, depressive symptoms, vital exhaustion, hopelessness); and medical measurements (body mass index, and systolic and diastolic blood pressure). Descriptive data on these variables are presented in Table 1.

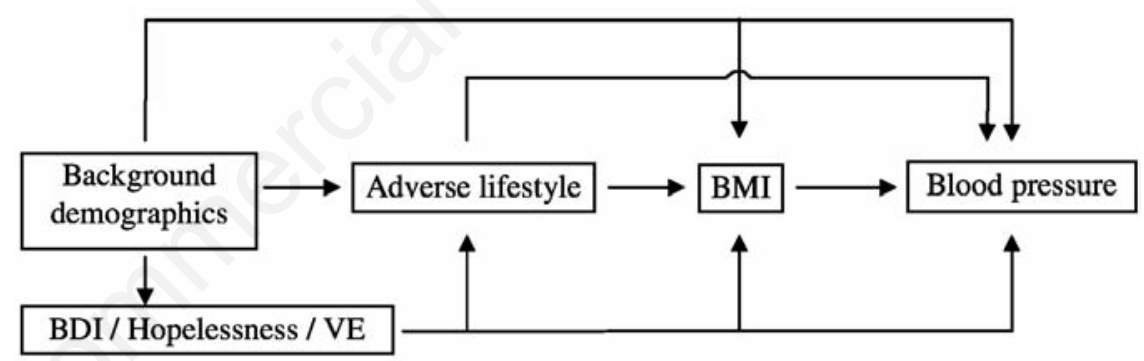

Figure 1. Model of the depressive symptoms/hopelessness/vital exhaustion - blood pressure relation.

Table 1. Psychological, lifestyle, medical and demographic characteristics of participants.

\begin{tabular}{lcccc} 
Variables & N & M & S.D. & Range \\
Depressive symptoms & 710 & 6.3 & 7.1 & $0-47$ \\
Hopelessness & 710 & 2.0 & 1.7 & $0-8$ \\
\hline Vital exhaustion & 710 & 9.7 & 9.7 & $0-42$ \\
Healthy food & 694 & 6.5 & 2.5 & $0-13$ \\
\hline Inactivity & 703 & 2.5 & 1.3 & $1-5$ \\
Smoking & 710 & 1.5 & 2.4 & $0-8$ \\
\hline Alcohol & 703 & 10.2 & 5.3 & $1-30$ \\
Coffee & 688 & 4.2 & 2.7 & $0-15$ \\
\hline BMI & 710 & 26.2 & 3.9 & $16.3-51.4$ \\
SBP (mmHg) & 710 & 130.3 & 15.5 & $96-208$ \\
\hline DBP (mmHg & 710 & 84.1 & 10.9 & $42-124$ \\
Age (years) & 710 & 49.4 & 3.8 & $45-56$ \\
\hline
\end{tabular}

Mean of diet variables represent the sum scores of the frequencies (per week) of consumption of specific aliments. The mean of inactivity represent the frequency of exercising per week. The mean of smoking represent the number of cigarettes per day. The mean of alcohol variable represents a product of frequency and quantity of alcohol consumption. A more complete description of these variables can be found in Measures. 
Education was assessed with a four-step question ( 1 = primary school, 2 = secondary school, 3 = high school, 4 = university degree).

Marital status was assessed with a single question that allowed differentiating among 4 groups: single, married/cohabitating, separated/divorced, widowed. For statistical analyses, this variable was dichotomised combining first, third, and fourth categories in one group, and keeping married people in a separate group ( $1=$ single/divorced/widowed, 2 = married/cohabitating).

Depressive symptoms were assessed using the 21-item Beck Depression Inventory (BDI). It has been widely used in assessment of psychological risks in relation with coronary artery disease.

Vital exhaustion was assessed using the 21item Maastricht Questionnaire. The scale has been previously used as a predictor of myocardial infarction.

Hopelessness was measured with a two-item Hopelessness Scale. Hopelessness has been shown to associate with the risk of CAD.

\section{Lifestyle factors}

Inactivity was assessed with one question about the frequency of exercising per week (1 $=3$ or more times per week, $2=1-2$ times per week, $3=1$ time per week, $4=$ from time to time, $5=$ not at all).

Smoking was evaluated with one question about number of cigarettes per day $(0=$ not at all, $1=$ from time to time, $2=1-4$ cigarettes per day, $3=5-9$ cigarettes per day, $4=10-14$ cigarettes per day, $5=15$-19 cigarettes per day, $6=20-24$ cigarettes per day, $7=25-29$ cigarettes per day, $8=30$ or more cigarettes per day).

Alcohol consumption was evaluated with two questions referring to the frequency of alcohol consumption $(1=$ not at all, $2=$ once or less per month, $3=2-4$ per month, $4=2-3$ per week, $5=4$ or more often per week) and quantity of alcohol consumed per drinking episode $(1=$ not at all, $2=1-2$ drinks, $3=3-4$ drinks, $4=5-6$ drinks, 5 = 7-9 drinks, $6=10$ or more drinks). Alcohol variable used in these analyses is the product of code numbers from questions of frequency and quantity of alcohol consumption.

Diet was evaluated with questions about how often a specific aliment was consumed per week ( $0=$ not at all, $1=1-2$ days, $2=3-5$ days, $3=6-7$ days). Healthy Food variable was calculated as sum scores of these answers for specific aliments consumed: muesli/cereals, rice/pasta, fish, fresh vegetables, fruits.

Coffee consumption variable evaluated how many cups of coffee were consumed each day.

\section{Medical variables}

Blood pressure was measured according to best practice at the time of the data collection. A single measurement was taken by the trained study nurses according to the standard protocol after at minimum of 30 minutes of rest. During the procedure the men were in sitting position, and the measurement was done using a calibrated mercury sphygmomanometer and the cuff around the right arm. The patients were asked not to smoke or drink coffee, tea or caffeine containing beverages or to do any heavy tasks at least 60 minutes before the measurement.

Body mass index was calculated by the standard formula: $\mathrm{kg} / \mathrm{m}^{2}$ (weight measured in kilograms was divided by squared height measured in meters).

\section{Statistical analyses}

Associations of depressive symptoms, hope- lessness and vital exhaustion with systolic BP (SBP) and diastolic BP (DBP) were analyzed using correlation analyses and path analyses. Statistical analyses were performed using the SPSS 14.0 software.

The hypothetical models of the predictors of BP were evaluated by using path analysis of the MPLUS version 5.2 software (http:// www.statmodel.com/download/usersguide/Mpl us\%20Users\%20Guide\%20v6.pdf). Before evaluation of path models, all missing data (number of missing cases presented in Table 1) were processed using the EM (expectationmaximization) algorithm available in SPSS 14.0 software. All factors in path analyses were used as continuous variables. Education, age and marital status were included in path analyses as possible confounding factors. Because distributions of depression, hopelessness and vital exhaustion were positively skewed, these variables were transformed for path analysis using root square procedure. For skewness and kurtosis the transformed variables had values below 1.0.

The chi-square test $\left(\chi^{2}\right)$, the root mean square error of approximation (RMSEA), the Tucker-Lewis fit index (TLI) and the comparative fit index (CFI) were fit indexes used to evaluate the model. The RMSEA value $<0.06$, TLI $>0.90$, CFI $>0.95$ and a non-significant $(P>0.05) \quad \chi^{2}$-test indicate an acceptable model..$^{20}$ Indirect mediation effects were tested using the bootstrapping procedure.

\section{Results}

The correlation matrix of all variables is presented in Table 2. Blood pressure values had no significant crude correlations with depres-

Table 2. Correlations of demographic variables, self-report measures and medical variables.

\begin{tabular}{|c|c|c|c|c|c|c|c|c|c|c|c|c|c|}
\hline & 2 & 3 & 4 & 5 & 6 & 7 & 8 & 9 & 10 & 11 & 12 & 13 & 14 \\
\hline Age & $-0.18^{\circ}$ & -0.01 & $0.12^{\circ}$ & $0.09 *$ & $0.08^{*}$ & $0.08^{*}$ & -0.01 & -0.03 & -0.03 & -0.40 & 0.01 & $0.15^{\circ}$ & -0.01 \\
\hline Education & & $0.10^{*}$ & $-0.14^{\circ}$ & $-0.19^{\circ}$ & $-0.12^{*}$ & $0.25^{\circ}$ & $-0.08 *$ & $-0.26^{\circ}$ & $-0.11 *$ & $-0.13^{\circ}$ & -0.03 & $-0.08^{*}$ & $-0.08 *$ \\
\hline Marital status & & & $-0.22^{\circ}$ & $-0.18^{\circ}$ & $-0.21^{\circ}$ & $0.11^{*}$ & $-0.10^{*}$ & $-0.15^{\circ}$ & $-0.09 *$ & 0.02 & 0.04 & 0.00 & 0.04 \\
\hline Depression & & & & $0.49^{\circ}$ & $0.84^{*}$ & $-0.16^{\circ}$ & $0.21^{\circ}$ & $0.17^{\circ}$ & $0.18^{\circ}$ & 0.05 & 0.05 & 0.00 & -0.03 \\
\hline Hopelessness & & & $0.44^{\circ}$ & & $0.45^{\circ}$ & $-0.18^{\circ}$ & $0.09 *$ & $0.10^{*}$ & 0.06 & -0.01 & -0.01 & -0.02 & -0.03 \\
\hline Vital exhaust. & & & $0.83^{\circ}$ & $0.39^{\circ}$ & & $-0.16^{\circ}$ & $0.21^{\circ}$ & $0.13^{\circ}$ & $0.18^{\circ}$ & 0.06 & 0.07 & 0.01 & 0.01 \\
\hline Healthy food & & & $-0.12^{\circ}$ & $-0.12^{\circ}$ & $-0.11^{\circ}$ & & $-0.24^{\circ}$ & $-0.32^{\circ}$ & $-0.18^{\circ}$ & $-0.16^{\circ}$ & -0.07 & $-0.12^{\circ}$ & $-0.15^{\circ}$ \\
\hline Inactivity & & & $0.19^{\circ}$ & 0.06 & $0.20^{\circ}$ & $-0.21^{\circ}$ & & $0.17^{\circ}$ & $0.09 *$ & 0.07 & $0.13^{\circ}$ & 0.03 & 0.06 \\
\hline Smoking & & & $0.12^{\circ}$ & 0.03 & 0.07 & $-0.23^{\circ}$ & $0.14^{\circ}$ & & $0.32^{\circ}$ & $0.30^{\circ}$ & -0.05 & -0.01 & $-0.09 *$ \\
\hline Alcohol & & & $0.15^{\circ}$ & 0.02 & $0.15^{\circ}$ & $-0.15^{\circ}$ & $0.10^{*}$ & $0.29^{\circ}$ & & 0.03 & 0.05 & $0.13^{\circ}$ & $0.16^{\circ}$ \\
\hline Coffee & & & 0.06 & -0.03 & 0.06 & $-0.12^{\circ}$ & 0.06 & $0.27^{\circ}$ & 0.03 & & $0.08^{*}$ & 0.01 & -0.02 \\
\hline BMI & & & 0.07 & -0.03 & $0.08^{*}$ & -0.07 & $0.14^{\circ}$ & -0.06 & 0.06 & $0.08 *$ & & $0.29^{\circ}$ & $0.34^{\circ}$ \\
\hline SBP & & & -0.03 & -0.05 & -0.01 & $-0.12^{\circ}$ & 0.03 & -0.03 & $0.12^{\circ}$ & 0.01 & $0.29^{\circ}$ & & $0.69^{\circ}$ \\
\hline DBP & & & -0.01 & -0.03 & 0.02 & $-0.13^{\circ}$ & 0.06 & $-0.11^{\circ}$ & $0.15^{\circ}$ & -0.03 & $0.34^{\circ}$ & $0.70^{\circ}$ & \\
\hline
\end{tabular}

${ }^{*} \mathrm{P}<0.05$. ${ }^{\circ} \mathrm{P}<0.01$ (2-tailed ). BMI, body mass index; SBP, systolic blood pressure; DBP, diastolic blood pressure. Above diagonal, correlations of all variables. Due to missing data N varies $675-710$. Below diagonal, correlations controlled for age, education and marital status, $\mathrm{N}=648$. 
sion, hopelessness or vital exhaustion. The strongest correlation was observed between depression and vital exhaustion scores (Table 2). Path analyses were used to investigate the relations between the study variables. Separate models for depression, hopelessness and vital exhaustion as main predictors of life styles and systolic and diastolic blood pressure were evaluated.

In our model the effect of depressive symptoms, hopelessness or vital exhaustion on blood pressure was supposed to work through lifestyle risk factors and body mass index as mediating factors. Further, independent direct links from depression, hopelessness and vital exhaustion to body mass index and blood pressure were expected to emerge as illustrated in the model presented in Figure 1. Education, age and marital status were controlled for as confounding factors.

In the present sample, $22.1 \%$ had primary school, and $28.9 \%$ secondary school, $25.4 \%$ had graduated high school, and $23.5 \%$ had graduated from university. Majority (77.7 \%) of the participants were married/cohabiting, and $22,3 \%$ were living alone. Fit indices for the models are presented in Table 3.

The path model of BDI and DBP had the same values as the path model of BDI and SBP (presented in Figure 2) except the following standardized solution coefficients, Age-DBP (not statistically significant), Alcohol-DBP (0.18), Smoking-DBP (-0.16), Healthy dietDBP (0.15), BMI-DBP (0.32), and the $\mathrm{R}^{2}$ value for DBP was 0.17.

BDI and VE path models with both systolic and diastolic BP were almost identical with only few minor exceptions: VE had no significant relationship with Smoking and Age, and the Education - VE standardized solution was 0.10 .

For hopelessness the path models indicated a relatively weak, albeit statistically significant direct inverse association with systolic BP (Figure 3); for diastolic BP the value of standardized coefficient $(-0.05)$ remained statistically non-significant. Furthermore, in hopelessness-diastolic BP path model, hopelessness and age had no significant relationship with diastolic BP. Also, there were some stronger standardized solution values, e.g. between diastolic BP and smoking $(-0.16)$, alcohol (0.17), healthy diet $(-0.16)$, and BMI (0.31). Consequently, the level of variance explained for diastolic BP was higher (0.17).

Fit indexes for all path models were excellent. The pattern of significant relations as well as the level of fit indexes for all of the models was to a great extent similar. The most significant exception was that hopelessness had a direct, inverse association with systolic blood pressure while for depression and vital exhaustion no significant direct impact on systolic or diastolic blood pressure was indicated.
Table 3. Fit indexes of the path analyses.

\begin{tabular}{lcccc} 
Model & $\chi^{2}$ & RMSEA & TH & CFI \\
BDI-SBP & $15.92(d f=11) \mathrm{P}=0.14$ & 0.025 & 0.95 & 0.99 \\
BDI-DBP & $16.24(d f=10) \mathrm{P}=0.09$ & 0.030 & 0.94 & 0.99 \\
\hline Hopelessness-SBP & $16.99(d f=11) \mathrm{P}=0.11$ & 0.028 & 0.99 & 0.95 \\
Hopelessness-DBP & $16.06(d f=10) \mathrm{P}=0.16$ & 0.010 & 0.94 & 0.99 \\
\hline VE-SBP & $15.27(d f=11) \mathrm{P}=0.17$ & 0.023 & 0.96 & 0.99 \\
VE-DBP & $18.79(d f=11) \mathrm{P}=0.07$ & 0.032 & 0.93 & 0.99 \\
\hline
\end{tabular}

$\chi^{2}$,chi-square test; RMSEA, root mean square error of approximation; TLI, Tucker-Lewis fit index; CFI, comparative fit index; BDI; Beck Depression Inventory; SBP, systolic blood pressure; DBP, diastolic blood pressure; VE, vital exhaustion.

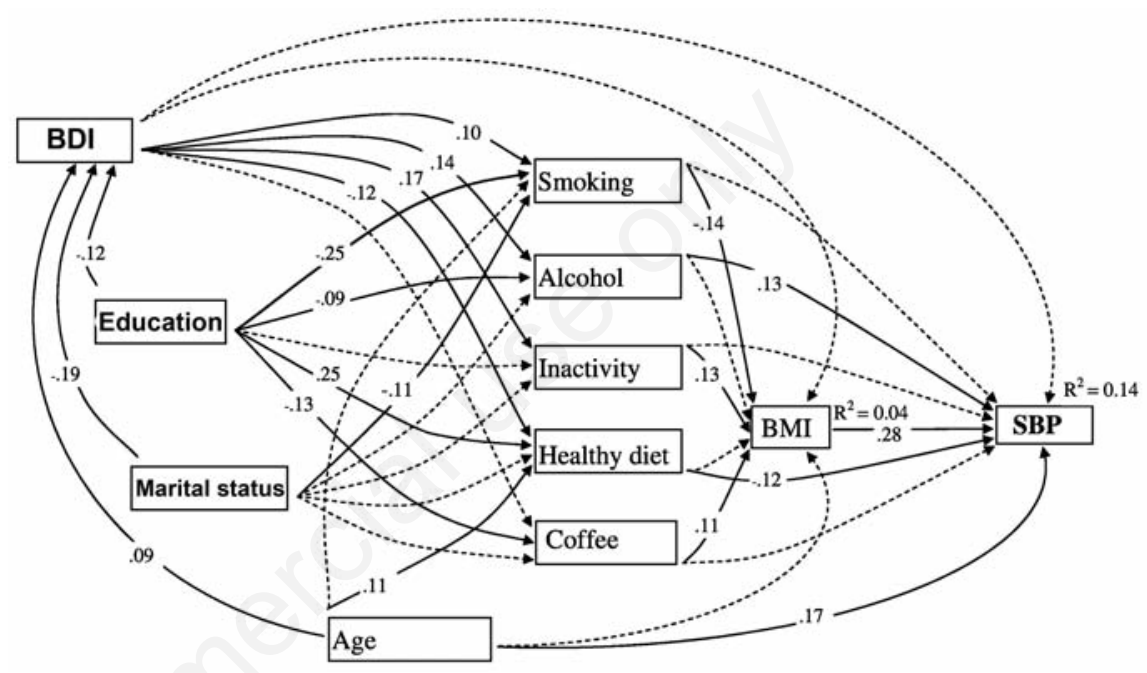

Figure 2. Significant (solid lines) and non-significant (dotted lines) relationships of the path model BDI - systolic BP; only significant coefficients are presented.

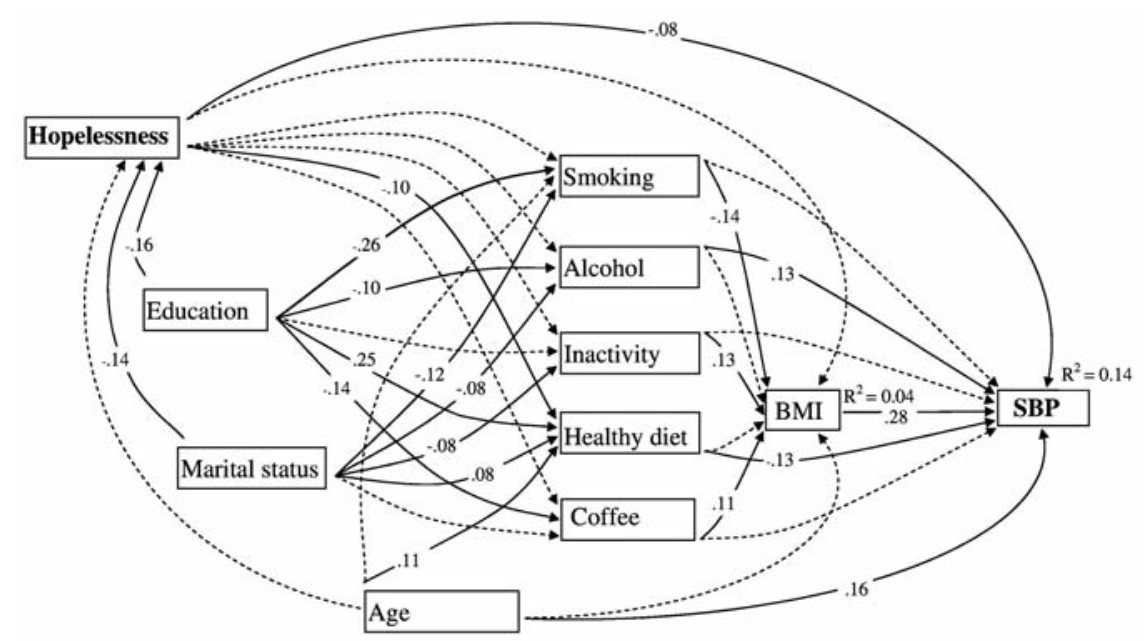

Figure 3. Significant (solid lines) and non-significant (dotted lines) relationships of the path model Hopelessness - systolic BP; only significant coefficients are presented. 
When testing all possible indirect mediation effects from depression to systolic and diastolic blood pressure there were significant positive indirect mediation effects from depression to diastolic blood pressure via alcohol consumption $\quad(\mathrm{B}=0.018, \quad \mathrm{P}<0.015$, bootstrap $\mathrm{CI}=0.003-0.032)$, via healthy diet $(\mathrm{B}=0.014$, $\mathrm{P}=0.042$, bootstrap $\mathrm{CI}=0.001-0.028$ ) and specific indirect effect via inactivity and BMI $(\mathrm{B}=0.005, \mathrm{P}=0.024$, bootstrap $\mathrm{CI}=0.001-0.010)$. Results were similar when examining the indirect effects from depression to systolic blood pressure. The only difference was that in this case the indirect mediation effect via healthy diet was only marginally significant $(B=0.011$, $\mathrm{P}=0.066$, bootstrap $\mathrm{CI}=-0.001-0.023)$. The indirect mediation effects between exhaustion and systolic or diastolic blood pressure were similar and have been presented in Table 4. Only significant indirect mediation effects from hopelessness to systolic or diastolic blood pressure via healthy diet were presented (Table 4).

\section{Discussion}

In this study we compared the associations of three depression-related constructs with health behavior and blood pressure. Results showed that two of them, depressive symptoms and vital exhaustion had very similar, significant associations with unfavorable lifestyle variables. Both of them were related to increased alcohol consumption, lack of exercise and a less healthy diet. The only exception seemed to be smoking, which related with depression, but not with exhaustion. At the same time, we found no direct association of depressive symptoms or vital exhaustion with blood pressure. Testing indirect mediation effects showed that alcohol consumption, diet, and inactivity could be possible mediators from depression or exhaustion to blood pressure. These results suggest that previously reported positive association between depression and exhaustion with blood pressure could be explained as mediated by adverse lifestyles. In one previous study it was also found that lifestyle factors but not depressive symptoms were associated with BP.21 Furthermore, their results seemed to exclude the possibility that lifestyle factors could act as mediators of the depression-hypertension relationship. Together with the present results this suggests that relations among these variables may be more complex than thought before. Clearly, the possible mediational role of health behavior in the depression-BP relationship needs more detailed investigation and especially studies based on longitudinal design.22

In contrast to the results with depressive symptoms and vital exhaustion, hopelessness revealed rather weak associations with health

Table 4. Standardized indirect mediation effects.

\begin{tabular}{|c|c|c|}
\hline \multirow[t]{2}{*}{ Indirect effect } & \multicolumn{2}{|c|}{ Dependent variable } \\
\hline & Systolic blood pressure & Diastolic blood pressure \\
\hline Exhaustion via alcohol & $0.174(0.004-0.033)^{*}$ & $0.025(0.007-0.043)^{*}$ \\
\hline Exhaustion via healthy diet & $0.014(0.001-0.028)^{\circ}$ & $0.018(0.002-0.033)^{\circ}$ \\
\hline Exhaustion via inactivity and BMI & $0.006(0.001-0.011)^{\circ}$ & $0.007(0.001-0.012)^{\circ}$ \\
\hline Hopelessness via alcohol & ns. & ns. \\
\hline Hopelessness via healthy diet & $0.013(0.001-0.025)^{\circ}$ & $0.015(0.002-0.029)^{\circ}$ \\
\hline Hopelessness via inactivity and BMI & ns. & ns. \\
\hline
\end{tabular}

behavior. Instead, a significant direct but inverse relationship between hopelessness and systolic BP was found. The negative relationship between hopelessness and diastolic BP was non-significant. The inverse relationship between hopelessness and systolic BP, corroborated with the absence of any significant relationships between BDI or VE and BP values, supports the argument that different depression-related constructs might work differently in context of health. This also supports the argument that hopelessness is to some extent a different concept from depression. 4

In their prospective study Everson et al.10 used the same two hopelessness questions as in the present one, and concluded that over a period of four years, initially normotensive men with high levels of hopelessness at baseline had a three-fold risk to become hypertensive. Despite using the same hopelessness measure and some common covariates in a middle-aged male sample, it is difficult to compare the contradictive results of these two studies. For example, the present sample included also hypertensive men, and hopelessness was at a lower level. In Everson's study the mean value of hopelessness was 2.4 , and those with high level of hopelessness represented $8.44 \%$ from their study sample while in our study the mean value of hopelessness was 1.96 , and those with high level of hopelessness represented only $4.1 \%$ from the sample. In line with this argumentation, results from another study, ${ }^{23}$ using the same hopelessness items and classes as Everson et al. found that high systolic BP was associated with high hopelessness and there was a significant mean difference of systolic BP between the three hopelessness groups. And again, those with high level of hopelessness represented a large percentage (34.5\%) from their study sample.

Furthermore, even Everson et al. found that those reporting moderate level of hopelessness were not at a significantly increased risk of hypertension. Possibly for the same reason, another study could not find any association between hopelessness and hypertension. ${ }^{24}$ One possible explanation for these results could be that there is a threshold effect in the association of hopelessness with BP, and a pos- itive association can be found only among the most hopeless subjects. This question remains for future studies to be answered.

The possible mechanisms through which hopelessness can cause hypertension include endothelial dysfunction or atherosclerosis. 25,26 Also this issue awaits further studies.

One finding that we did not expect was the inverse relation of smoking with diastolic BP, because it is well known that smoking produces a chronic increase of BP for active smokers. ${ }^{27}$ However, previous literature displays several contradictive findings on the smoking and BP relation. Studies have found that smoking increases $\mathrm{BP}, 27$ but also found no relation between smoking and BP. ${ }^{28}$ Even more, some studies suggested that smoking is related to a decrease in BP or that giving up smoking is related to higher BP.28,29 One explanation of these contradictory findings could be related to complex interactions of the smoking behavior with lifestyles and health. BP is influenced by BMI and,30 through higher energy expenditure and lower appetite, smoking can lead to a lower BMI. ${ }^{31}$ In our sample, smoking had statistically significant inverse relations with BMI and also with diastolic BP. The direct inverse relationship of smoking with BP could be explained through some not yet fully understood physiological mechanisms. One study found arguments that genotypic variations influence the relationship between smoking status and the probability to develop hypertension. 32 Another report suggests that smoking behavior is related to higher BP only if smoking induces degenerative changes or remodeling of small arteries. ${ }^{33}$

The main limitations of this study were related to the cross-sectional nature of the survey and reliance on self-reports of depressive symptoms as well as of lifestyle data. Furthermore, in the present study only middleaged men were included. Future studies should include women and larger variation of age as well as prospective designs.

Furthermore, despite the numerous statistically significant paths in our models, the amount of explained variance of blood pressure was only about $14-17 \%$ indicating that some important determinants of blood pres- 
sure could not be assessed in this study. One could also argue that using only single BP measurement weakens the reliability of our dependent variable. However, because most of our analyses are based on correlations, and considering the findings presented in the Journal of Hypertension, ${ }^{34}$ a double or triple measurement of BP would have probably changed only marginally the results of this study. Most likely our results are actually underestimates of true associations.

In this study, symptoms of depression were assessed with BDI because this scale has been the most widely used method in studies of CVD risk factors, offering validated reference values, and it has been shown in numerous studies to be a reliable and valid instrument to assess symptoms of depression. Also, the prevalence of depression observed in this study is comparable to larger population studies conducted with standard interview methods. ${ }^{35}$ However, the use of this scale contains a potential limitation because it has been originally developed for psychiatric patients. In future studies, the use of other scales developed for medical patients should be considered.

Assessment of nutrition in this study was based on the frequency of consuming certain aliments and therefore our measure should be considered only as a tentative estimate of healthy nutrition.

Finally, because this study was based on a cross-sectional database and engaged statistical techniques from which no directionality can be suggested, the causal role of any variables can be only speculated.

At the same time, there are several strengths in our study. First, this study was based on a large sample of healthy individuals that allows for a powerful statistical testing. Second, it compared the importance of different facets of depression (depressive symptoms, vital exhaustion, and hopelessness) as related to blood pressure. Third, to our knowledge, this study is the first to investigate the role of different health behaviors as mediators in the relationship between facets of depression and blood pressure with thorough control of confounding variables such as age, education, and marital status.

\section{Conclusions}

As a conclusion, in this study of middle-aged men, the results indicate that vital exhaustion and depressive symptoms relate with several unfavorable lifestyles, but have no direct relationship with BP values. Together with the observed indirect effects these results suggest that previously reported relations of depression and vital exhaustion with BP could have been mediated by unfavorable lifestyles. Hopelessness is not so closely related with adverse health behaviors as depression or vital exhaustion, and it had an inverse association with systolic BP. Results of this study do not support the importance of hopelessness as a psychological risk factor for elevated blood pressure.

\section{References}

1. Suls J, Bunde J. Anger, anxiety, and depression as risk factors for cardiovascular disease: the problems and implications of overlapping affective dispositions. Psychol Bull 2005;131:260-300.

2. Lett HS, Blumenthal JA, Babyak MA, et al. Depression as a risk factor for coronary artery disease: evidence, mechanisms, and treatment. Psychosom Med 2004;66: 305-15.

3. Appels A. Mental precursors of myocardial infarction. Br J Psychiatry 1990;156:46571.

4. Everson SA, Goldberg DE, Kaplan GA, et al. Hopelessness and risk of mortality and incidence of myocardial infarction and cancer. Psychosom Med 1996;58:113-21.

5. Kubzansky LD, Kawachi I. Going to the heart of the matter: do negative emotions cause coronary heart disease? J Psychosom Res 2000;48:323-37.

6. Valtonen M, Laaksonen DE, Laukkanen J, et al. Leisure-time physical activity, cardiorespiratory fitness and feelings of hopelessness in men. BMC Public Health 200925;9:204.

7. Scalco AZ, Scalco MZ, Azul JB, Lotufo Neto F. Hypertension and depression. Clinics (Sao Paulo) 2005;60:241-50.

8. Joiner TE Jr, Steer RA, Abramson LY, et al. Hopelessness depression as a distinct dimension of depressive symptoms among clinical and non-clinical samples. Behav Res Ther 2001;39:523-36.

9. Abela JR. The hopelessness theory of depression: a test of the diathesis-stress and causal mediation components in third and seventh grade children. J Abnorm Child Psychol 2001;29:241-54.

10. Everson SA, Kaplan GA, Goldberg DE, Salonen JT. Hypertension incidence is predicted by high levels of hopelessness in Finnish men. Hypertension 2000;35:561-7.

11. Kopp MS, Falger PR, Appels A, Szedmak S. Depressive symptomatology and vital exhaustion are differentially related to behavioral risk factors for coronary artery disease. Psychosom Med 1998;60:752-8.

12. Lahlou-Laforet K, Alhenc-Gelas M, Pornin M, et al. Relation of depressive mood to plasminogen activator inhibitor, tissue plasminogen activator, and fibrinogen levels in patients with versus without coronary heart disease. Am J Cardiol 2006;97:1287-91.

13. Wojciechowski FL, Strik JJ, Falger P, et al. The relationship between depressive and vital exhaustion symptomatology postmyocardial infarction. Acta Psychiatr Scand 2000;102:359-65.

14. Schwartz SW, Carlucci C, Chambless LE, Rosamond WD. Synergism between smoking and vital exhaustion in the risk of ischemic stroke: evidence from the ARIC study. Ann Epidemiol 2004;14:416-24.

15. Conduit E, Appels A, Lewis A. Cardioprotective effect of moderate drinking: possible mediation by vital exhaustion. Alcohol Alcohol 1998;33:528-32.

16. Brezinka V, Dusseldorp E, Maes S. Gender differences in psychosocial profile at entry into cardiac rehabilitation. J Cardiopulm Rehabil 1998;18:445-9.

17. Koertge JC, Ahnve S, Schenck-Gustafsson $\mathrm{K}$, et al. Vital exhaustion in relation to lifestyle and lipid profile in healthy women. Int J Behav Med 2003;10:44-55.

18. Joyner-Grantham J, Mount DL, McCorkle OD, et al. Self-reported influences of hopelessness, health literacy, lifestyle action, and patient inertia on blood pressure control in a hypertensive emergency department population. Am J Med Sci 2009;338:368-72.

19. Igna CV, Julkunen J, Vanhanen H. Anger expression styles and blood pressure: evidence for different pathways. J Hypertens 2009;27:1972-9.

20. Kline RB. Principles and practice of structural equation model. New York: Guilford Press; 2005. p 446.

21. Reiff M, Schwartz S, Northridge M. Relationship of depressive symptoms to hypertension in a household survey in Harlem. Psychosom Med 2001;63:711-21.

22. Spencer SJ, Zanna MP, Fong GT. Establishing a causal chain: why experiments are often more effective than mediational analyses in examining psychological processes. J Pers Soc Psychol 2005;89: 845-51.

23. Valtonen M, Laaksonen DE, Tolmunen T, et al. Hopelessness -- novel facet of the metabolic syndrome in men. Scand J Public Health 2008;36:795-802.

24. Stern SL, Dhanda R, Hazuda HP. Helplessness predicts the development of hypertension in older Mexican and European Americans. J Psychosom Res 2009;67:333-7.

25. Do DP, Dowd JB, Ranjit N, et al. Hopelessness, depression, and early markers of endothelial dysfunction in U.S. adults. Psychosom Med 2010;72:613-9.

26. Pollitt RA, Daniel M, Kaufman JS, et al. 
Mediation and modification of the association between hopelessness, hostility, and progression of carotid atherosclerosis. $\mathrm{J}$ Behav Med 2005;28:53-64.

27. Narkiewicz K, Kjeldsen SE, Hedner T. Is smoking a causative factor of hypertension? Blood Press 2005;14:69-71.

28. Okubo Y, Suwazono Y, Kobayashi E, Nogawa K. An association between smoking habits and blood pressure in normotensive Japanese men: a 5-year followup study. Drug Alcohol Depend 2004;73:167-74.

29. Lee DH, Ha MH, Kim JR, Jacobs DR Jr. Effects of smoking cessation on changes in blood pressure and incidence of hyper- tension: a 4-year follow-up study. Hypertension 2001;37:194-98.

30. Brock CM, King DS, Wofford MR, Harrell TK. Exercise, insulin resistance, and hypertension: a complex relationship. Metab Syndr Relat Disord 2005;3:60-5.

31. Chiolero A, Faeh D, Paccaud F, Cornuz J. Consequences of smoking for body weight, body fat distribution, and insulin resistance. Am J Clin Nutr 2008;87:801-9.

32. Abe M, Nakura J, Yamamoto M, et al. Association of GNAS1 gene variant with hypertension depending on smoking status. Hypertension 2002;40:261-5.

33. Takashima Y, Yoshida M, Ishikawa M, et al. Interrelations among smoking habits, casual blood pressure and intraocular pressure in middle and old-aged japanese residents. Environ Health Prev Med 2002;7:162-8.

34. Boveta P, Gervasonia J, Rossa AG, et al. Assessing the prevalence of hypertension in populations: are we doing it right? J Hypertension 2003;21:509-17.

35. Pirkola SP, Isometsa E, Suvisaari J, et al. DSM-IV mood-, anxiety- and alcohol use disorders and their comorbidity in the Finnish general population--results from the Health 2000 Study. Soc Psychiatry Psychiatr Epidemiol 2005;40:1-10. 